Mathematical Problems in Mechanics

\title{
A note on statistical solutions of the three-dimensional Navier-Stokes equations: The time-dependent case
}

\section{Note sur les solutions statistiques des équations de Navier-Stokes incompressibles en dimension trois d'espace : le cas dépendant du temps}

\author{
Ciprian Foias ${ }^{\mathrm{a}}$, Ricardo M.S. Rosa ${ }^{\mathrm{b}}$, Roger Temam ${ }^{\mathrm{c}, \mathrm{d}}$ \\ a Department of Mathematics, Texas A\&'M University, College Station, TX 77843, USA \\ b Instituto de Matemática, Universidade Federal do Rio de Janeiro, Caixa Postal 68530 Ilha do Fundão, Rio de Janeiro, RJ 21945-970, Brazil \\ c Académie des Sciences, Paris \\ ${ }^{\mathrm{d}}$ Department of Mathematics, Indiana University, Bloomington, IN 47405, USA
}

\section{A R T I C L E I N F O}

\section{Article history:}

Received 7 December 2009

Accepted 29 December 2009

Available online 20 February 2010

Presented by Philippe G. Ciarlet

\begin{abstract}
A B S T R A C T
Time-dependent statistical solutions of the three-dimensional Navier-Stokes equations for incompressible fluids are considered. They are a mathematical formalization of the notion of ensemble averages in turbulence theory and form the backbone for a mathematical foundation of the theory of turbulence. The two main notions of statistical solutions, previously introduced, are revisited and a new formulation of one of them is given. An existence proof for this new formulation is given, along with a number of useful properties.

(c) 2010 Published by Elsevier Masson SAS on behalf of Académie des sciences.

R É S U M É

Dans cette Note nous considérons les solutions statistiques des équations de NavierStokes incompressibles en dimension trois d'espace. Elles constituent une formalisation mathématique de la notion de moyenne statistique dans la théorie de la turbulence et forment l'un des fondements de la théorie mathématique de la turbulence. Deux notions différentes de solutions statistiques ont été introduites; nous les rappelons et donnons une formulation nouvelle de l'une d'elles. Nous établissons en outre un théorème d'existence de solutions pour cette nouvelle notion, et donnons un certain nombre de propriétés utiles des solutions statistiques.
\end{abstract}

(c) 2010 Published by Elsevier Masson SAS on behalf of Académie des sciences.

\section{Version française abrégée}

Dans cette Note nous nous intéressons à la formulation mathématique des solutions statistiques des équations de NavierStokes incompressibles en dimension trois d'espace. Les solutions statistiques ont été introduites pour formaliser la notion de moyenne statistique qui se trouve au centre de la théorie conventionnelle de la turbulence [1,8-12,14-19]. Notre objet ici est de revoir et comparer les formulations mathématiques des deux notions de solutions statistiques qui ont été introduites par Foias et Prodi [2,3], et par Vishik et Fursikov [21] respectivement.

E-mail addresses: rrosa@ufrj.br (R.M.S. Rosa), temam@indiana.edu (R. Temam). 
Une solution statistique est définie en [2,3] comme une famille de mesures paramétrisées par la variable de temps et, à chaque instant la mesure est associée à une distribution statistique du champ des vitesses. Par ailleurs les solutions statistiques sont définies en [21] comme une mesure sur l'espace des trajectoires. En s'inspirant de cette dernière définition, nous introduisons le concept de mesures de Vishik-Fursikov qui sont essentiellement des mesures portées par l'ensemble des solutions faibles dites de Leray-Hopf, et qui satisfont une certaine inégalité d'énergie.

Nous rappelons qu'une solution faible de Leray-Hopf des équations de Navier-Stokes est une solution faible au sens habituel, qui satisfait en outre une certaine inégalité d'énergie rappelée dans le texte (voir (2.2)), et qui est fortement continue à l'instant initial dans l'espace de base $H$ qui est défini comme la fermeture dans $L^{2}(\Omega)^{3}$ de l'espace $\mathcal{V}$ (voir après $(2.1)$ ). Cette inégalité d'énergie et la continuité a l'instant initial jouent un rôle essentiel dans la définition des solutions statistiques au point que l'on est amené à faire la différence entre les espaces de trajectoires $\mathcal{U}_{I}$ et $\mathcal{U}_{I}^{\#}$ définis en (2.3), (2.4), qui ne diffèrent que par la continuité à l'origine. Les solutions de Leray-Hopf sont aussi faiblement continues dans $\mathrm{H}$ à tout instant, ce qui nous conduit naturellement à faire un usage systématique de la topologie faible de $H$ et à considérer les mesures définies sur la tribut des ensemble Boréliens de $H$ muni de sa topologie faible. Ceci nous permet d'utiliser un certain nombre de propriétés fines en théorie de la mesure.

La définition que nous donnons ici des mesures de Vishik-Fursikov est à comparer à la définition initiale donnée en [21], à savoir celle d'une mesure sur l'ensemble des trajectoires qui satisfont une inégalité d'énergie moins précise et qui est portée par des sous ensembles mesurables de l'espace des solutions faibles qui ne sont pas nécessairement du type de Leray-Hopf.

En projetant à chaque instant une mesure de Vishik-Fursikov sur l'espace des phases, nous obtenons une solution statistique particulière que l'on appelle ici solution statistique de Vishik-Fursikov. Cette classe de solutions statistiques se prête beaucoup mieux à un traitement analytique que les solutions statistiques définies en [2], et ces solutions statistiques possèdent un certain nombre de propriétés utiles. En contre partie, et en raison du caractère minimal des conditions imposées pour définir les solutions statistiques de Vishik-Fursikov, la démonstration de l'existence d'une mesure de Vishik-Fursikov et du fait que la famille de ses projections à chaque instant est une solution statistique est assez délicate. Pour cette raison, nous ne pouvons pas donner ici l'idée des démonstrations et renvoyons le lecteur intéressé à un article à paraitre [6].

Une propriété intéressante que nous établissons est relative au support d'une mesure de Vishik-Fursikov. Nous prouvons essentiellement que la continuité de l'énergie cinétique en moyenne à l'instant initial implique la continuité à l'instant initial presque partout pour les solutions déterministes correspondantes, presque partout au sens de cette mesure. Nous donnons aussi une caractérisation intrinsèque des solutions statistiques de Vishik-Fursikov comme limite de combinaisons convexes de mesures de Dirac portées par les valeurs à chaque instant d'un ensemble fini de solutions faibles de Leray-Hopf.

Nous considérons ici les solutions statistiques des équations de Navier-Stokes qui dépendent du temps; le cas stationnaire sera considéré dans [5]. Tous les détails et toutes les démonstrations pour les deux cas seront donnés dans [6,7].

\section{Introduction}

We consider the mathematical formulation of statistical solutions of the three-dimensional Navier-Stokes equations for incompressible fluids. Statistical solutions have been introduced to formalize the notion of ensemble average, so pervasive in the conventional theory of turbulence. Recently, a number of rigorous results have been obtained in turbulence theory based on this concept. Our aim is to reconsider the mathematical formulation of the two main definitions of statistical solutions, introduced by Foias and Prodi [2,3] and Vishik and Fursikov [21], respectively.

A statistical solution as defined in $[2,3]$ is a family of measures parametrized by the time variable and associated with the probability distribution of the velocity field of the flow at each time. The definition given in [21], on the other hand, was of a single measure on the space of trajectories. Inspired by their definition we introduce the concept of a Vishik-Fursikov measure, which is essentially that of a measure carried by the set of Leray-Hopf weak solutions and satisfying a mean energy inequality.

We recall that a Leray-Hopf weak solution is a weak solution in the classical sense of the Navier-Stokes equations which also satisfies a certain energy inequality and is strongly continuous at the initial time. This energy inequality and the strong continuity at the origin play a crucial role in the formulations of statistical solutions. Leray-Hopf weak solutions are also weakly continuous at any given time, a fact that led us quite naturally to make extensive use of the weak topology and the associated Borel measures, on the $L^{2}$-related $H$ space, allowing for the use of a number of results in measure theory which are, however, quite delicate.

The definition given here of a Vishik-Fursikov measure is to be compared with the original definition in [21], which was of a measure on the space of trajectories satisfying a less sharp mean energy inequality and carried by measurable subsets of the space of weak solutions not necessarily of Leray-Hopf type.

Projecting a Vishik-Fursikov measure to the phase space at each time gives a particular type of statistical solution which we term a Vishik-Fursikov statistical solution. This class of statistical solutions is much more amenable to analysis than that in [2] and possesses a number of useful properties. On the other hand, and partly because of the minimal requirements in the definition of a Vishik-Fursikov statistical solution, the proofs of existence of a Vishik-Fursikov measure and that the family of their projections at each time is a statistical solution are quite involved. For this reason, we present here the main results but omit their proofs. 
Another significant result that we obtain is related to the localization of the carrier of a Vishik-Fursikov measure, giving essentially that the continuity of the kinetic energy at the initial time in the mean implies the continuity at the initial time almost everywhere with respect to the measure. We also give an intrinsic characterization of Vishik-Fursikov statistical solutions as limits of convex combinations of Dirac measures carried by the values at each given time of finite collections of Leray-Hopf weak solutions.

Here we only consider time-dependent statistical solutions. Stationary statistical solutions will be considered in [5]. More details on both cases will be given in $[6,7]$.

\section{Preliminaries}

\subsection{The Navier-Stokes equations and the mathematical setting}

We consider the incompressible Navier-Stokes equations in space dimension three. We consider two cases: flows on an open, bounded, regular set $\Omega$ in $\mathbb{R}^{3}$ with no-slip boundary conditions (Dirichlet conditions); and periodic flows in $\mathbb{R}^{3}$ with periods $L_{1}, L_{2}, L_{3}$ in each direction $0 x_{1}, 0 x_{2}, 0 x_{3}$, respectively, and in this case we set $\Omega$ to be the period $\Omega=\Pi_{i=1}^{3}\left(0, L_{i}\right)$.

We recall that in either case the equations can be given the following concise functional formulation [20,13]:

$$
\frac{\mathrm{d} \mathbf{u}}{\mathrm{dt}}+v A \mathbf{u}+B(\mathbf{u}, \mathbf{u})=\mathbf{f}
$$

in the space $H$. This space is defined as the completion in $L^{2}(\Omega)^{3}$ of

$$
\mathcal{V}_{\text {Dir }}=\left\{\mathbf{u} \in \mathcal{C}_{\mathrm{c}}^{\infty}\left(\mathbb{R}^{3}\right)^{3} ; \nabla \cdot \mathbf{w}=0\right\}
$$

in the Dirichlet case, and

$$
\mathcal{V}_{\text {per }}=\left\{\mathbf{u}=\left.\mathbf{w}\right|_{\Omega} ; \begin{array}{l}
\mathbf{w} \in \mathcal{C}^{\infty}\left(\mathbb{R}^{3}\right)^{3}, \nabla \cdot \mathbf{w}=0, \int_{\Omega} \mathbf{w}(\mathbf{x}) \mathrm{d} \mathbf{x}=0, \mathbf{w}(\mathbf{x}) \text { is periodic with period } L_{i} \\
\text { in each direction } 0 x_{i}
\end{array}\right\}
$$

in the periodic case.

We also consider the space $V$ which is the completion of either $\mathcal{V}_{\text {Dir }}$ or $\mathcal{V}_{\text {per }}$ in $H^{1}(\Omega)^{3}$, depending on the case. The spaces $H$ and $V$ are endowed with the usual inner products

$$
(\mathbf{u}, \mathbf{v})=\int_{\Omega} \mathbf{u}(\mathbf{x}) \cdot \mathbf{v}(\mathbf{x}) \mathrm{d} \mathbf{x}, \quad((\mathbf{u}, \mathbf{v}))=\int_{\Omega} \sum_{i=1,2,3} \frac{\partial \mathbf{u}}{\partial x_{i}} \cdot \frac{\partial \mathbf{v}}{\partial x_{i}} \mathrm{~d} \mathbf{x},
$$

and the associated norms $|\mathbf{u}|=(\mathbf{u}, \mathbf{u})^{1 / 2},\|\mathbf{u}\|=((\mathbf{u}, \mathbf{u}))^{1 / 2}$.

The forcing term $\mathbf{f}$ is assumed to time independent and to belong to $H$.

We denote by $P_{\mathrm{LH}}$ the (Leray-Helmholtz) orthogonal projector in $L^{2}(\Omega)^{3}$ onto the subspace $H$. In the Dirichlet case, the operator $A$ in (1) is the Stokes operator given by $A \mathbf{u}=-P_{\mathrm{LH}} \Delta \mathbf{u}$, for $\mathbf{u} \in D(A)$, with domain $D(A)=V \cap H^{2}(\Omega)^{3}$. In the periodic case, $A$ is given by $A \mathbf{u}=-P_{\mathrm{LH}} \Delta \mathbf{u}=-\Delta \mathbf{u}$, for $\mathbf{u} \in D(A)$, with the domain $D(A)$ as the closure in $H^{2}(\Omega)^{3}$ of $\mathcal{V}_{\text {per }}$.

Finally, the term $B(\mathbf{u}, \mathbf{v})=P_{\mathrm{LH}}((\mathbf{u} \cdot \nabla) \mathbf{v})$ is a bilinear term associated with the inertial term $(\mathbf{u} \cdot \nabla) \mathbf{u}$ of the Navier-Stokes equations.

\subsection{Weak solutions}

We call a function $\mathbf{u}=\mathbf{u}(t)$ on a time interval $I \subset \mathbb{R}$ with values in $H$ a (Leray-Hopf) weak solution of (1) on $I$ if (i) $\mathbf{u} \in L_{\text {loc }}^{\infty}(I ; H) \cap L_{\text {loc }}^{2}(I ; V)$; (ii) $t \mapsto \mathbf{u}(t)$ is weakly continuous in $H$; (iii) $\mathbf{u}$ satisfies the functional equation (1) in the distribution sense on $I$, with values in the dual $V^{\prime}$ of $V$; (iv) for almost all $t^{\prime}$ in $I$, u satisfies the energy inequality

$$
\frac{1}{2}|\mathbf{u}(t)|^{2}+v \int_{t^{\prime}}^{t}\|\mathbf{u}(s)\|^{2} \mathrm{~d} s \leqslant \frac{1}{2}\left|\mathbf{u}\left(t^{\prime}\right)\right|^{2}+\int_{t^{\prime}}^{t}(\mathbf{f}, \mathbf{u}(s)) \mathrm{d} s,
$$

for all $t$ in $I$ with $t>t^{\prime}$; and (v) if $I$ is closed and bounded on the left, with left end point denoted by $t_{0}$, then $\mathbf{u}$ is strongly continuous in $H$ at $t_{0}$ from the right, i.e. $\mathbf{u}(t) \rightarrow \mathbf{u}\left(t_{0}\right)$ in $H$ as $t \rightarrow t_{0}^{+}$. The times $t^{\prime}$ allowed in (2) are the points of strong continuity from the right for $\mathbf{u}$, and their set is of total measure.

We recall that for any interval $I=\left[t_{0}, \infty\right)$ and any $\mathbf{u}_{0} \in H$, there exists a Leray-Hopf weak solution on $I$ such that $\mathbf{u}(0)=\mathbf{u}_{0}$. 


\subsection{Trajectory spaces}

We define some basic "time-dependent" function spaces. In what follows, we let $I$ be an arbitrary interval in $\mathbb{R}$ and let $H_{\mathrm{w}}$ denote the space $H$ endowed with its weak topology. We consider the space $\mathcal{C}\left(I, H_{\mathrm{w}}\right)$ of continuous functions from $I$ into $H_{\mathrm{w}}$ endowed with the topology of uniform weak convergence on compact intervals in $I$.

For each $t_{0} \in I$, we define the projection operators $\Pi_{t_{0}}: \mathcal{C}\left(I, H_{\mathrm{w}}\right) \rightarrow H_{\mathrm{w}}$ by $\Pi_{t_{0}} \mathbf{u}=\mathbf{u}\left(t_{0}\right)$. Denoting by $I^{\circ}$ the interior of $I$, we also define the spaces of weak solutions

$$
\begin{aligned}
& \mathcal{U}_{I}=\left\{\mathbf{u} \in \mathcal{C}\left(I, H_{\mathrm{w}}\right) ; \mathbf{u} \text { is a weak solution on } I\right\}, \\
& \mathcal{U}_{I}^{\sharp}=\left\{\mathbf{u} \in \mathcal{C}\left(I, H_{\mathrm{w}}\right) ; \mathbf{u} \text { is a weak solution on } I^{\circ}\right\},
\end{aligned}
$$

endowed with the topologies inherited from $\mathcal{C}\left(I, H_{\mathrm{w}}\right)$. The spaces $\mathcal{U}_{I}$ and $\mathcal{U}_{I}^{\sharp}$ are separable Hausdorff locally convex topological vector spaces. The space $\mathcal{U}_{I}^{\sharp}$ is $\sigma$-compact, i.e. it is a countable union of compact spaces. In general, $\mathcal{U}_{I} \subset \mathcal{U}_{I}^{\sharp}$, and if $I$ is open on the left, then $\mathcal{U}_{I}=\mathcal{U}^{\sharp}$, while if $I$ is closed and bounded on the left, then $\mathcal{U}_{I}$ is a dense $\mathcal{G}_{\delta \sigma}$-set in $\mathcal{U}_{I}^{\sharp}$. Notice the subtlety in the definitions of $\mathcal{U}_{I}$ and $\mathcal{U}_{I}^{\sharp}$, in which the only difference is when the interval $I$ is closed and bounded on the left, for which functions in $\mathcal{U}_{I}$ are necessarily strongly continuous in $H$ at the left end point, while in $\mathcal{U}_{I}^{\sharp}$ they may only be weakly continuous.

Remark 2.1. We give here an example to illustrate the difference between $\mathcal{U}_{I}$ and $\mathcal{U}_{I}^{\sharp}$. Consider a sequence $\left\{\mathbf{u}_{n}\right\}_{n}$ of weak solutions of (1) on an interval $[0, T), T>0$, and belonging to $\mathcal{U}_{[0, T)}$, with $\mathbf{f}=\mathbf{f}_{n}$ and $\mathbf{u}_{n}(0)=\mathbf{u}_{0 n}$. If $\mathbf{f}_{n}$ converges weakly to $\mathbf{f}$ in $H$ and $\mathbf{u}_{0 n}$ converges weakly to $\mathbf{u}_{0}$ in $H$, it is well known that the limit $\mathbf{u}$ of $\mathbf{u}_{n}$ exists on $[0, T)$ and $\mathbf{u}$ is a weak solution of (1) on the open interval $(0, T)$. This limit belongs to $\mathcal{U}_{[0, T)}^{\sharp}$ but if $\mathbf{u}_{0 n}$ converges weakly to $\mathbf{u}_{0}$ but not strongly, then one cannot so far guarantee that $\mathbf{u} \in \mathcal{U}_{[0, T)}$.

\section{Time-dependent statistical solutions}

In this section we consider the definition and properties of time-dependent statistical solutions and of Vishik-Fursikov measures of the Navier-Stokes equations, casting a new perspective on the theories given in earlier works [2,21] (see also $[4,22])$.

For the definition of statistical solutions one needs to consider appropriate test functions. For this purpose we consider the cylindrical test functions, namely functionals $\Phi: H \rightarrow \mathbb{R}$ of the form

$$
\Phi(\mathbf{u})=\phi\left(\left(\mathbf{u}, \mathbf{v}_{1}\right), \ldots,\left(\mathbf{u}, \mathbf{v}_{k}\right)\right),
$$

where $k \in \mathbb{N}, \phi$ is a $C^{1}$ real-valued function on $\mathbb{R}^{k}$ with compact support, and $\mathbf{v}_{1}, \ldots, \mathbf{v}_{k}$ belong to $V$. For such $\Phi$, we denote by $\Phi^{\prime}$ its Fréchet derivative in $H$.

Then, a (time-dependent) statistical solution is defined as follows:

Definition 3.1. For a given interval $I \subset \mathbb{R}$, a family $\left\{\mu_{t}\right\}_{t \in I}$ of Borel probability measures on $H$ is called a statistical solution of the Navier-Stokes equations on $I$ if the following conditions hold:

(i) The function $t \mapsto \int_{H} \Phi(\mathbf{u}) \mathrm{d} \mu_{t}(\mathbf{u})$ is measurable on $I$ for every bounded and continuous real-valued function $\Phi$ on $H$;

(ii) The functions $t \mapsto \int_{H}|\mathbf{u}|^{2} \mathrm{~d} \mu_{t}(\mathbf{u})$ and $t \mapsto \int_{H}\|\mathbf{u}\|^{2} \mathrm{~d} \mu_{t}(\mathbf{u})$ belong to $L_{\mathrm{loc}}^{\infty}(I)$ and $L_{\text {loc }}^{1}(I)$, respectively;

(iii) For any cylindrical test function $\Phi$, the Liouville-type equation

$$
\int_{H} \Phi(\mathbf{u}) \mathrm{d} \mu_{t}(\mathbf{u})=\int_{H} \Phi(\mathbf{u}) \mathrm{d} \mu_{t^{\prime}}(\mathbf{u})+\iint_{t^{\prime}}^{t}\left(\mathbf{f}, \Phi^{\prime}(\mathbf{u})\right)-v\left(\left(\mathbf{u}, \Phi^{\prime}(\mathbf{u})\right)\right)-\left(B(\mathbf{u}, \mathbf{u}), \Phi^{\prime}(\mathbf{u})\right) \mathrm{d} \mu_{s}(\mathbf{u}) \mathrm{d} s
$$

holds for all $t^{\prime}, t \in I$;

(iv) A strengthened mean energy inequality holds on $I$, i.e. for any nonnegative, nondecreasing, continuously-differentiable real-valued function $\psi:[0, \infty) \rightarrow \mathbb{R}$ with bounded derivative, there exists a set $I^{\prime} \subset I$ of full measure on $I$ such that

$$
\begin{aligned}
& \frac{1}{2} \int_{H} \psi\left(|\mathbf{u}|_{0}^{2}\right) \mathrm{d} \mu_{t}(\mathbf{u})+v \int_{t^{\prime}}^{t} \int_{H} \psi^{\prime}\left(|\mathbf{u}|^{2}\right)\|\mathbf{u}\|^{2} \mathrm{~d} \mu_{s}(\mathbf{u}) \mathrm{d} s \\
& \quad \leqslant \frac{1}{2} \int_{H} \psi\left(|\mathbf{u}|^{2}\right) \mathrm{d} \mu_{t^{\prime}}(\mathbf{u})+\iint_{t^{\prime} H}^{t} \psi^{\prime}\left(|\mathbf{u}|^{2}\right)(\mathbf{f}, \mathbf{u}) \mathrm{d} \mu_{s}(\mathbf{u}) \mathrm{d} s
\end{aligned}
$$

holds for any $t^{\prime} \in I^{\prime}$ and for all $t \in I$ with $t \geqslant t^{\prime}$; and 
(v) If $I$ is closed and bounded on the left with left end point $t_{0}$, then the function

$$
t \mapsto \int_{H} \psi\left(|\mathbf{u}|^{2}\right) \mathrm{d} \mu_{t}(\mathbf{u})
$$

is continuous at $t=t_{0}$ from the right, for every function $\psi$ as in (iv).

In (iv), the set $I^{\prime}$, which may depend on $\psi$, is the set of points $t^{\prime}$ where the function (7) is continuous from the right. Given an initial Borel probability measure $\mu_{0}$ on $H$ with finite mean kinetic energy, i.e. satisfying

$$
\int_{H}|\mathbf{u}|^{2} \mathrm{~d} \mu_{0}(\mathbf{u})<\infty,
$$

there exists a time-dependent statistical solution $\left\{\mu_{t}\right\}_{t} \geqslant t_{0}$ with $\mu_{t_{0}}=\mu_{0}$. Such an existence result of time-dependent statistical solutions was first proved in [2, Theorem 1, p. 254, and Proposition 1, p. 291] via Galerkin approximation.

In the approach of Vishik and Fursikov [22], the statistical solutions are obtained from probability measures carried by the family of all individual weak solutions. With that in mind we have the following definitions:

Definition 3.2. A Vishik-Fursikov measure over an interval $I \subset \mathbb{R}$ is defined as a Borel probability measure $\rho$ on the space $\mathcal{C}\left(I, H_{\mathrm{W}}\right)$ which is carried by $\mathcal{U}_{I}^{\sharp}$ and such that $t \rightarrow \int_{\mathcal{U}_{I}^{\sharp}}|\mathbf{u}(t)|^{2} \mathrm{~d} \rho(\mathbf{u}) \in L_{\text {loc }}^{\infty}(I)$. Moreover, if $I$ is closed and bounded on the left, with left end point $t_{0}$, then for any nonnegative, nondecreasing continuously-differentiable real-valued function $\psi:[0, \infty) \rightarrow \mathbb{R}$ with bounded derivative, we have

$$
\lim _{t \rightarrow t_{0}^{+}} \int_{\mathcal{U}_{I}^{\sharp}} \psi\left(|\mathbf{u}(t)|^{2}\right) \mathrm{d} \rho(\mathbf{u})=\int_{\mathcal{U}_{I}^{\sharp}} \psi\left(\left|\mathbf{u}\left(t_{0}\right)\right|^{2}\right) \mathrm{d} \rho(\mathbf{u})<\infty .
$$

Definition 3.3. A Vishik-Fursikov statistical solution of the Navier-Stokes equations on an interval $I \subset \mathbb{R}$ is a statistical solution $\left\{\rho_{t}\right\}_{t \in I}$ such that $\rho_{t}=\Pi_{t} \rho$, for all $t \in I$, for some Vishik-Fursikov measure $\rho$ over the interval $I$.

In their original work [22], the authors only consider measures similar to the Vishik-Fursikov measures given in Definition 3.2. Since the above definitions of Vishik-Fursikov measure and Vishik-Fursikov statistical solutions are different from the original definition in [22], the existence of these objects requires a new proof. Our first result concerns then the existence of Vishik-Fursikov measures.

Theorem 3.1. Let $t_{0} \in \mathbb{R}$ and let $\mu_{0}$ be a Borel probability measure on $H$ with finite mean kinetic energy (i.e. satisfying (8)). Then, there exists a Vishik-Fursikov measure $\rho$ over the time interval $\left[t_{0}, \infty\right)$ such that $\Pi_{t_{0}} \rho=\mu_{0}$.

Then, some work is needed to show that the projection of a Vishik-Fursikov measure is a statistical solution.

Theorem 3.2. Let $I \subset \mathbb{R}$. If $\rho$ is a Vishik-Fursikov measure over the interval $I$, then the family of projections $\left\{\rho_{t}\right\}_{t \in I}$ is a statistical solution on I (in the sense of Definition 3.1).

Theorems 3.1 and 3.2 directly imply that given $t_{0} \in \mathbb{R}$ and a Borel probability measure $\mu_{0}$ on $H$ satisfying $\int_{H}|\mathbf{u}|^{2} \mathrm{~d} \mu_{0}(\mathbf{u})<\infty$, there exists a Vishik-Fursikov statistical solution $\left\{\rho_{t}\right\}_{t \geqslant t_{0}}$ over the interval $\left[t_{0}, \infty\right)$ satisfying $\rho_{t_{0}}=\mu_{0}$. Clearly this is an improvement of the existence theorem in $[2,3]$.

Although a Vishik-Fursikov measure over an interval $I$ is defined as being carried by a set of functions which are weak solutions on the interior $I^{\circ}$, it is remarkable that it turns out to be carried by the collection of weak solutions on the whole $I$. This follows, in particular, from the continuity in the mean at the initial time. More precisely, we have the following result:

Theorem 3.3. Let $\rho$ be a Vishik-Fursikov measure over an interval $I \subset \mathbb{R}$. Then $\rho\left(\mathcal{U}_{I}^{\sharp} \backslash \mathcal{U}_{I}\right)=0$, and hence $\rho$ is carried by $\mathcal{U}_{I}$.

Because of the minimal requirements in the definition of a Vishik-Fursikov measure the proofs of the above results are long and laborious and hard to sketch, therefore we omit them. They will be given in [6]. 


\section{Characterization of the Vishik-Fursikov statistical solutions}

A natural question is when a statistical solution in the sense of Definition 3.1 is a Vishik-Fursikov statistical solution. In this section, we give an answer to this question for statistical solutions which have bounded (uniformly in time) support in $H$ and when the time interval is open; namely, we present an intrinsic characterization of Vishik-Fursikov statistical solutions which belong to that class.

Let $I \subset \mathbb{R}$ be an interval and let $R>0$. Let $\operatorname{co} \mathcal{D}_{R}$ denote the set of convex combinations of (deterministic) statistical solutions of the form $\left\{\delta_{\mathbf{u}(t)}\right\}_{t \in I}$, where $\mathbf{u}$ is a weak solution on $I$ satisfying $|\mathbf{u}(t)| \leqslant R$, for all $t \in I$. Any such statistical solution is in fact a Vishik-Fursikov statistical solution, with associated Vishik-Fursikov measure given as the convex combination of measures $\left\{\delta_{\mathbf{u}}\right\}$.

Theorem 4.1. Let $I \subset \mathbb{R}$ be an open interval, $R>0$ be given, and let $\left\{\mu_{t}\right\}_{t \in I}$ be a statistical solution of the Navier-Stokes equations satisfying $\mu_{t}\left(B_{H}(R)\right)=1$, for all $t \in I$, where $B_{H}(R)$ is the ball in $H$ of radius $R$ and centered at the origin. Then, $\mu$ is a Vishik-Fursikov statistical solution if and only if there exists a sequence of statistical solutions $\left\{\mu_{t}^{(n)}\right\}_{t \in I} \in \operatorname{co} \mathcal{D}_{R}, n \in \mathbb{N}$, such that, for any real-valued weakly-continues function $\varphi$ on $B_{H}(R)$,

$$
\int_{t} \varphi(\mathbf{u}) \mathrm{d} \mu_{t}^{(n)}(\mathbf{u}) \rightarrow \int_{t} \varphi(\mathbf{u}) \mathrm{d} \mu_{t}(\mathbf{u}), \quad \text { as } n \rightarrow \infty,
$$

uniformly in $t$ for $t$ in any compact subset of $I$.

The proof of this result will be given in [6].

\section{References}

[1] G.K. Batchelor, The Theory of Homogeneous Turbulence, Cambridge University Press, Cambridge, 1953.

[2] C. Foias, Statistical study of Navier-Stokes equations I, Rend. Sem. Mat. Univ. Padova 48 (1972) 219-348.

[3] C. Foias, G. Prodi, Sur les solutions statistiques des équations de Navier-Stokes, Ann. Mat. Pura Appl. 111 (4) (1976) 307-330.

[4] C. Foias, O.P. Manley, R. Rosa, R. Temam, Navier-Stokes Equations and Turbulence, Encyclopedia of Mathematics and Its Applications, vol. 83, Cambridge University Press, 2001.

[5] C. Foias, O.P. Manley, R. Rosa, R. Temam, A note on statistical solutions of the three-dimensional Navier-Stokes equations: the stationary case, C. R. Acad. Sci. Paris, Ser. I 348 (2010), doi:10.1016/j.crma.2009.12.018.

[6] C. Foias, R. Rosa, R. Temam, Properties of time-dependent statistical solutions of the three-dimensional Navier-Stokes equations, in preparation.

[7] C. Foias, R. Rosa, R. Temam, Properties of stationary statistical solutions of the three-dimensional Navier-Stokes equations, in preparation.

[8] U. Frisch, Turbulence, The Legacy of A.N. Kolmogorov, Cambridge University Press, Cambridge, 1995. xiv+296 pp.

[9] J.O. Hinze, Turbulence, McGraw-Hill, New York, 1975.

[10] E. Hopf, Statistical hydromechanics and functional calculus, J. Ration. Mech. Anal. 1 (1952) 87-123.

[11] A.N. Kolmogorov, The local structure of turbulence in incompressible viscous fluid for very large Reynolds numbers, C. R. (Dokl.) Acad. Sci. USSR (N.S.) 30 (1941) 301-305.

[12] A.N. Kolmogorov, On degeneration of isotropic turbulence in an incompressible viscous liquid, C. R. (Dokl.) Acad. Sci. USSR (N.S.) 31 (1941) $538-540$.

[13] J. Leray, Etude de diverses équations intégrales non linéaires et de quelques problèmes que pose l'hydrodynamique, J. Math. Pures Appl. 12 (1933) $1-82$.

[14] M. Lesieur, Turbulence in Fluids, 3rd ed., Fluid Mechanics and Its Applications, vol. 40, Kluwer Academic Publishers Group, Dordrecht, 1997, xxxii+515 pp.

[15] A.S. Monin, A.M. Yaglom, Statistical Fluid Mechanics: Mechanics of Turbulence, MIT Press, Cambridge, MA, 1975.

[16] A.M. Obukhoff, On the energy distribution in the spectrum of turbulent flow, C. R. (Dokl.) Acad. Sci. USSR 32 (1941) 19-21.

[17] O. Reynolds, On the dynamical theory of incompressible viscous fluids and the determination of the criterion, Phil. Trans. Roy. Soc. London A 186 (1895) 123-164.

[18] G.I. Taylor, Statistical theory of turbulence, Proc. Roy. Soc. London Ser. A 151 (1935) 421-478.

[19] G.I. Taylor, The spectrum of turbulence, Proc. Roy. Soc. London Ser. A 164 (1938) 476-490.

[20] R. Temam, Navier-Stokes Equations and Nonlinear Functional Analysis, 2nd ed., SIAM, Philadelphia, 1995.

[21] M.I. Vishik, A.V. Fursikov, L'équation de Hopf, les solutions statistiques, les moments correspondant aux systémes des équations paraboliques quasilinéaires, J. Math. Pures Appl. 59 (9) (1977) 85-122.

[22] M.I. Vishik, A.V. Fursikov, Mathematical Problems of Statistical Hydrodynamics, Kluwer, Dordrecht, 1988. + Additional references. 